DOI: https://doi.org/10.11144/Javeriana.umed62-2.emed

\title{
Educación médica basada en competencias: revisión de enfoque
}

\section{Competency-Based Medical Education: Scoping Review}

Recibido: 27 Noviembre 2020 | Aceptado: 24 Enero 2021

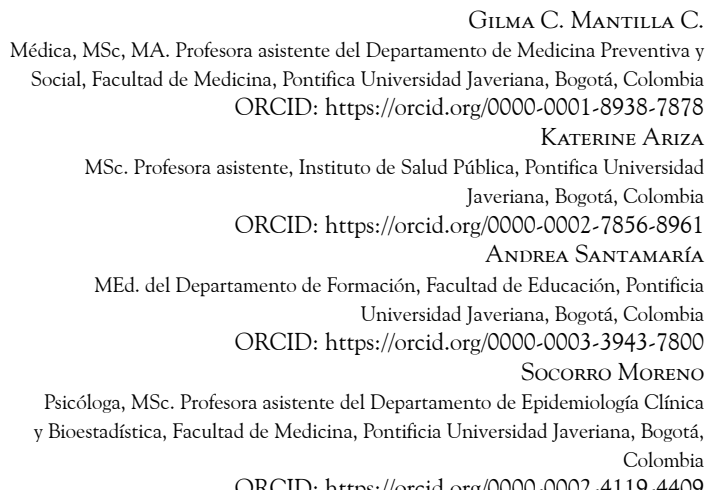

Gilma C. Mantilla C.

Médica, MSc, MA. Profesora asistente del Departamento de Medicina Preventiva y Social, Facultad de Medicina, Pontifica Universidad Javeriana, Bogotá, Colombia ORCID: https://orcid.org/0000-0001-8938-7878 Katerine Ariza

MSc. Profesora asistente, Instituto de Salud Pública, Pontifica Universidad Javeriana, Bogotá, Colombia ORCID: https://orcid.org/0000-0002-7856-8961 ANDREa Santamaría MEd. del Departamento de Formación, Facultad de Educación, Pontificia Universidad Javeriana, Bogotá, Colombia ORCID: https://orcid.org/0000-0003-3943-7800

SOCORRo MOREnO

Psicóloga, MSc. Profesora asistente del Departamento de Epidemiología Clínica y Bioestadística, Facultad de Medicina, Pontificia Universidad Javeriana, Bogotá, Colombia

Cómo citar: Mantilla GC, Ariza K, Santamaría A, Moreno S. Educación médica basada en competencias: revisión de enfoque. Univ. Med. 2021;62(2). https://doi.org/10.11144/Javeriana.umed 62-2.emed

\section{RESUMEN}

Introducción: El modelo de educación basada en competencias es cada vez más frecuente para la formación de pregrado en medicina; sin embargo, falta consenso sobre el concepto de competencia, que puede limitar la comprensión y el análisis de los programas curriculares que lo emplean. Objetivo: Analizar las definiciones y aplicaciones de los términos currículo basado en competencias (CBC) y evaluación basada en competencias (EvBC) en la literatura de educación médica para proponer un marco conceptual en los programas de pregrado de medicina. Método: Revisión de enfoque con búsquedas en seis bases de datos. Publicaciones en el tema de la evaluación por competencias o CBC en el área médica, específicamente en los programas de pregrado, durante las últimas tres décadas. Resultados: De 3618 artículos, se preseleccionaron 89 para revisión completa. Se incluyeron 34 artículos. El análisis cualitativo generó 4 categorías principales y 14 categorías subordinadas. Conclusiones: Aunque no existe un consenso que defina la competencia, CBC y EvBC, la decisión de implementar un CBC en un programa médico de pregrado implica el reconocimiento del contexto social y regulatorio, así como un desarrollo progresivo que responda a las necesidades particulares que pueden estar relacionadas con todos los niveles del aprendizaje.

Palabras clave

educación basada en competencias; educación de pregrado en medicina; educación médica; evaluación educacional; aprendizaje.

\begin{abstract}
Introduction: The competency-based education model is increasingly common for undergraduate medicine training. However, there is a lack of consensus on the concept of competence that can limit the understanding and analysis of the curricular programs that use it. Objective: To analyze the definitions and applications of the terms competency-based curriculum
\end{abstract}


(CBC) and competency-based assessment (EvBC) in the medical education literature to propose a conceptual framework in undergraduate medicine programs. Method: Scoping review with searches in six databases. Publications about competency assessment or CBC in the medical area, specifically in undergraduate programs, during the last three decades. Results: Of 3618 articles, 89 were preselected for full review. 34 articles were included. The qualitative analysis generated 4 main categories and 14 subordinate categories. Conclusions: Although there is no consensus that defines competence, $\mathrm{CBC}$ and $\mathrm{EvBC}$, the decision to implement a $\mathrm{CBC}$ in an undergraduate medical program implies recognition of the social and regulatory context and a progressive development that responds to particular needs, which may be related to all levels of learning.

Keywords

competency-based education; undergraduate medical education; medical education; educational assessment; learning.

\section{Introducción}

El desarrollo de currículos basados en competencias (CBC), en las diferentes áreas del conocimiento, responde a las demandas laborales y a las exigencias del mundo actual, cada vez más mediatizado por la sociedad de la información y el conocimiento. A partir de estas orientaciones se vienen adelantado diversos cambios en las universidades, que apuntan a transformaciones conceptuales sobre la finalidad de la formación de profesionales, el perfil de impacto social y la respuesta a una misión universitaria que se preocupe más por el sentido integral de la formación humana y que aporte a la construcción de ciudadanos del mundo. Lo anterior se hace evidente en las diferentes reformas curriculares para que los programas académicos se adapten a las exigencias legales y a las demandas sociales.

La educación basada en competencias se ha hecho más frecuente en la educación médica alrededor del mundo (1). Frank et al. (2) llevaron a cabo una revisión sistemática en 2010 sobre sus definiciones, en la que llegaban a la siguiente propuesta:

La educación basada en competencias (EBC) es un enfoque para preparar médicos para la práctica que está fundamentalmente orientado a las habilidades de resultados de los graduados y se organiza en torno a competencias derivadas de un análisis de las necesidades sociales y de los pacientes. No hace hincapié en la capacitación basada en el tiempo y promete una mayor responsabilidad, flexibilidad y concentración en el alumno. (p. 636)

Esta definición tiene algunos conceptos implícitos (competencias, evaluación por competencias y $\mathrm{CBC}$ ), cuyas definiciones, sin embargo, no fueron cubiertas por la revisión. En el mismo sentido, el documento de educación médica basada en competencias (EMBC) de la Asociación de Facultades de Medicina de Canadá, presenta los componentes clave de la EMBC, el Marco de Van Melle, en el que las competencias y la evaluación por competencias tienen un rol fundamental (1), aunque no presenta definiciones operacionales que permitan accionar la planeación educativa con mayor claridad.

La Facultad de Medicina de la Pontificia Universidad Javeriana, sede Bogotá, en 2014 inició el proceso de implementación de su plan curricular basado en competencias con el fin de responder a los desafíos actuales de la educación médica en Colombia, entre estos: mejorar la integración entre áreas del conocimiento, establecer un sistema de formación basado en el desarrollo de competencias profesionales y responder adecuadamente a las necesidades del país. En este marco se desarrolla un proyecto de investigación titulado La actividad integradora como herramienta para el desarrollo de competencias profesionales en estudiantes de medicina, cuyo propósito es identificar si la actividad integradora puede ser considerada una herramienta $y$ estrategia de estudio interdisciplinar, que haga posible el desarrollo y dominio de las competencias que los profesionales médicos requieren para su formación académica y práctica en diferentes contextos.

En el marco de dicho proyecto, se lleva a cabo esta revisión de enfoque, cuyo objetivo se orientó a explorar y sintetizar los diversos significados que la literatura en educación médica ha dado a los conceptos de competencias, evaluación basada en competencias (EvBC) y $\mathrm{CBC}$, pues la comprensión de los conceptos implícitos en la EMBC es fundamental para llevar a cabo los 
ajustes tanto en la planeación educativa como en el análisis de los programas desarrollados en el interior de nuestro plan curricular.

\section{Método}

Empleamos las guías sugeridas por el Joanna Briggs Institute (3) y por Thomas et al. (4), para conducir una revisión de enfoque sobre las expresiones currículo basado en competencias y evaluación basada en competencias dentro de la literatura de educación médica. Como parte de esta revisión, entendimos que no hay una definición unívoca sobre el término competencias para aplicarlo en las reformas o lineamientos curriculares en programas de pregrado de medicina, ya que hay diferentes aproximaciones teóricas que no han sido consensuadas y, por lo tanto, su aplicación en guías internacionales ha dependido fuertemente del contexto.

\section{Pregunta de investigación}

Nuestra revisión de enfoque se centró en responder la pregunta: icómo se entienden los términos competencia, evaluación por competencias . currículo basado en competencias en las propuestas curriculares de programas de pregrado de Medicina? Después de revisar los antecedentes, encontramos que los términos competencias y evaluación, a menudo, se complementan con currículo y aprendizaje; por lo tanto, decidimos incluir estos términos en nuestro análisis.

\section{Identificación de estudios relevantes}

Utilizamos los siguientes indicadores booleanos (currículum $\mathrm{O}$ currículo $\mathrm{O}$ currícula $\mathrm{O}$ medicine program $\mathrm{O}$ competency-based education $\mathrm{O}$ competency-based evaluation) Y (medicine $\mathrm{O}$ medical students . medical education) para nuestra búsqueda en las bases de datos PubMed y Embase (filtradas a resultados relevantes de ERIC, WorldCat y Búsqueda Académica Completa). La búsqueda inicial fue el 12 de febrero de
2018 y una búsqueda de artículos de seguimiento ocurrió el 22 de febrero de 2018. Además, debido a nuestra ubicación en Colombia y al hecho de que algunas de las bases de datos más grandes no incluyen publicaciones locales o de región, decidimos buscar directamente en las bases de datos Latindex, Scopus y ProQuest y EBSCOhost. En ambos casos, limitamos los resultados por fecha de publicación a los últimos treinta años; el idioma a inglés, español y portugués, y el nivel exclusivamente de pregrado en medicina, excluyendo aquellos que tuvieran un enfoque de residencia, maestría o doctorado.

\section{Selección de los estudios relevantes}

Todas las referencias las importamos al software Mendeley y eliminamos los duplicados. Dos de nosotras (SM y AS) aplicamos una herramienta de selección a todos los títulos y resúmenes de artículos recuperados para determinar su elegibilidad.

Para una revisión completa del artículo, comprobamos todos los títulos y resúmenes no duplicados para su inclusión. Después de esta revisión, organizamos una matriz de elegibilidad en Excel, que depuramos cinco veces con base en la relación título-indicadores booleanos, resumen-indicadores booleanos, resumen-título, revisión-título, currículo-título y currículoresumen, lo que nos permitió identificar qué artículos incluir para una revisión completa.

A continuación, cada una de las investigadoras leímos un número designado de los artículos seleccionados, para una revisión exhaustiva a través del diligenciamiento de un formato de resumen analítico educativo. Ello llevó a que, bajo estrictos criterios de selección, eligiéramos un número de artículos que todas las investigadoras íbamos a leer. Para ser incluido en la hoja de extracción de datos, cada artículo necesitaba:

1. Enfocarse y nombrar explícitamente evaluación por competencias, reforma curricular y competencias, desarrollo de competencias o el concepto de 
competencia en la educación médica en pregrado.

2. Involucrar los planes de estudio de los programas de pregrado de medicina en el ámbito internacional con base en el modelo de educación basada en competencias.

Es necesario aclarar que excluimos libros, reseñas de libros, comentarios y cartas al editor. Para confirmar el rigor del proceso de selección, buscamos en las bibliografías de los artículos seleccionados otras fuentes directas citadas por ellos que incluyeran el concepto de competencia en educación médica.

\section{Extracción de datos}

Para la extracción de datos, resumimos la información de los artículos seleccionados y registramos los datos en una hoja de Excel (Microsoft, Redmond, Washington), para analizarlos a través de un marco común compuesto por datos como: a) demográficos e identificación (año de publicación, autores, revista de publicación), b) datos de contenido (título, resumen, objetivos, diseño metodológico y conclusiones) y c) categorías temáticas (competencias, evaluación, currículo y aprendizaje). El proceso de revisión fue iterativo, es decir, agregamos y editamos columnas en nuestra hoja de cálculo según la necesidad a lo largo del proceso.

\section{Resultados}

La figura 1 presenta el diagrama de búsqueda, la selección y la inclusión final de artículos para la presente revisión.

\section{Figura 1}

Diagrama PRISMA de la búsqueda (5)

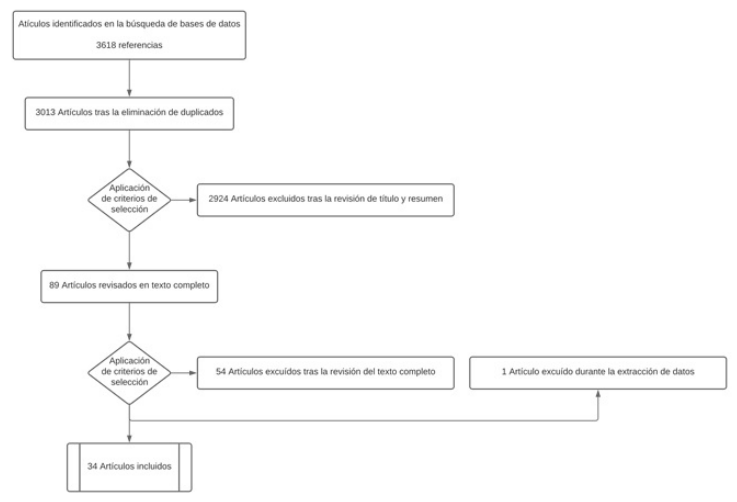

La tabla 1 presenta las características generales de los documentos incluidos; mientras que la tabla 2, la clasificación según el tipo de artículo de los documentos que conforman esta revisión.

\section{Tabla 1}

Características de los estudios incluidos $(n=34)$

\begin{tabular}{|c|c|c|}
\hline Característica & Núm. & Porcentaje \\
\hline \multicolumn{3}{|c|}{$\begin{array}{l}\text { Fecha de publicación } \\
\end{array}$} \\
\hline 1990-1999 & 6 & 18 \\
\hline $2000-2010$ & 25 & 74 \\
\hline $2011-2018$ & 3 & 9 \\
\hline \multicolumn{3}{|c|}{ País de origen } \\
\hline Estados Unidos & 17 & 50 \\
\hline Reino Unido & 6 & 18 \\
\hline Australia & 4 & 12 \\
\hline Arabia Saudita & 1 & 3 \\
\hline Canadá & 1 & 3 \\
\hline Países Bajos & 2 & 6 \\
\hline Escocia & 1 & 3 \\
\hline México & 1 & 3 \\
\hline Argentina & 1 & 3 \\
\hline \multicolumn{3}{|c|}{ Tipo de publicación } \\
\hline Artículo científico & 3 & 9 \\
\hline Artículo de divulgación & 8 & 24 \\
\hline Reporte de resultados & 8 & 24 \\
\hline Guías & 7 & 21 \\
\hline Revisión teórica & 8 & 21 \\
\hline
\end{tabular}


Tabla 2.

Tipo de artículos incluidos en la revisión $(n=34)$

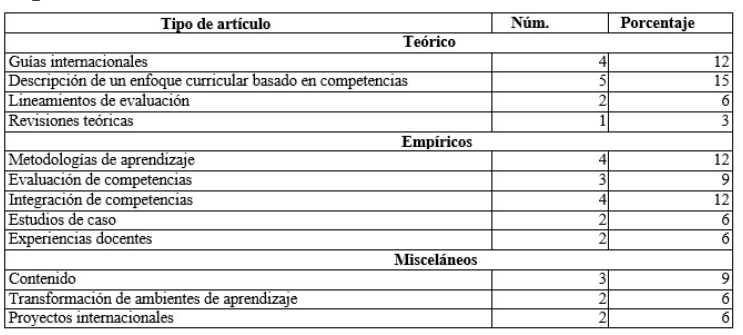

A continuación, se presentan los resultados por cada una de las categorías de análisis.

\section{Competencias}

De los 34 artículos revisados, el 62,9\% aborda el concepto de competencias; de estos, el 31,8 $\%$ plantea definiciones y el 40,9\% identifica las competencias en el ámbito médico. Por su parte, el 13,6\% define qué son las habilidades y el restante 13,6\% las describe. Las competencias a nivel general se definen como el desempeño efectivo y eficiente de una función, de un papel o de una posición que integra un conjunto de conocimientos, destrezas, habilidades y actitudes en el desarrollo de tareas y actividades. Por ello, las competencias se componen tanto de los conocimientos y habilidades como de las actitudes y valores necesarios para la ejecución de tareas, la solución de problemas y el desempeño eficaz en una determinada profesión, posición o rol (6).

Miller desarrolló un modelo para definir la competencia profesional organizando una pirámide de cuatro niveles. En los dos niveles de la base de la pirámide se sitúan los conocimientos (saber qué) y cómo aplicarlos a casos concretos (saber cómo). En el nivel inmediatamente superior (mostrar cómo) se ubica la competencia cuando es medida en ambientes in vitro (simulados) $\mathrm{y}$ donde el profesional debe demostrar todo lo que es capaz de hacer. En la cima se halla el desempeño (saber hacer) o lo que el profesional realmente lleva a cabo en la práctica real, independientemente de lo que demuestra que es capaz de hacer (7).
En el marco de un CBC, deben establecerse criterios claros para su identificación y creación. La literatura revisada indica que en la educación superior existen competencias genéricas - comunes a todas las profesiones que son transversales al proceso de formación - y competencias específicas —referidas a los campos profesionales particulares que se desarrollan en los cursos y asignaturas- (8). En el caso de las competencias en programas de pregrado de medicina, estas deberán: 1) enfocarse en las metas y en el desempeño esperado del médico cuando se gradúe, 2) reflejar las expectativas del programa, 3) ser expresadas en términos medibles y 4) emplear un estándar claro para su medición (9).

Las competencias médicas se han definido de diferentes formas: algunos autores las entienden como el conjunto de comportamientos basados en los componentes de conocimiento, destreza, actitudes y habilidades personales observables e importantes para la práctica de la medicina (9); otros, como la adquisición de un conocimiento de base fuerte y amplio, que se suma a un rango de destrezas clínicas y profesionales, además de unos comportamientos ejemplares profesionales y humanísticos (10), los cuales incluyen actitudes y valores esenciales para el ejercicio de la medicina $(11,12,13,14,15,16,17)$.

En medicina, también se han identificado tres grandes tipos de competencias: las conceptuales, las clínicas y las culturales. Las primeras se desarrollan a través de la comprensión profunda de los principios generales del dominio, que permiten aplicar los conocimientos en diferentes contextos, así como adaptarlos a tareas novedosas. Las competencias clínicas aluden a la habilidad para obtener datos del paciente a través de la historia clínica y el examen físico, que luego se integran a la impresión diagnóstica y al manejo eficaz del paciente. Por último, las competencias culturales involucran los comportamientos, los conocimientos, las actitudes y las políticas para el trabajo efectivo en diversos contextos y situaciones (10). En los artículos revisados, identificamos diferentes tipos de competencias que se presentan en la tabla 3. 
Tabla 3

Organización de las competencias en la formación médica

\begin{tabular}{|c|c|c|}
\hline Competencias & Tipo de saber & Expresión de la competencia \\
\hline Disciplinares & $\begin{array}{l}\text { Saber qué } \\
\text { Saber hacer }\end{array}$ & 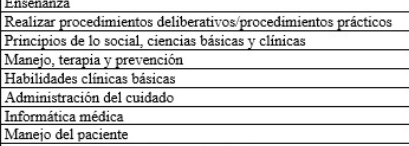 \\
\hline Interdisciplinares & Saber cómo & 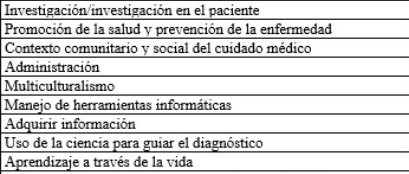 \\
\hline Metadisciplinares & Saber ser & 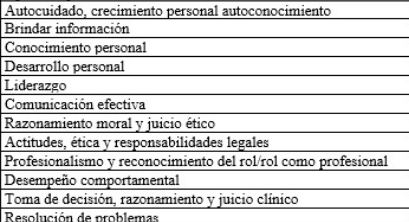 \\
\hline
\end{tabular}

Fuente: elaboración propia a partir de $(11,12,13,14,15)$.

\section{Currículo}

De los 34 artículos revisados, el 80,0\% hace referencia a la categoría currículo, y de esta, el $82,1 \%$, a aspectos curriculares, y el $17,9 \%$, a reforma curricular. El currículo se entiende como una agrupación de intenciones, expectativas, contenidos, estrategias y experiencias educativas, resultados de aprendizaje, evaluación de procesos formales e informales, en los cuales los estudiantes ganan conocimiento y comprensión, desarrollan habilidades y destrezas, modifican sus actitudes y valores bajo el apoyo del equipo docente y directivo en el marco de una cultura educativa institucional $(10,18)$.

La planeación del currículo es el eje central de todos los aspectos curriculares que se consideran en una reforma de este tipo (19). Esta planeación debe contemplar la definición de objetivos, dominios, métodos de enseñanza-aprendizaje, estrategias pedagógicas y tipos de evaluación (20). Estos componentes deben estar siempre enmarcados en alguna o varias teorías de la cognición, como la cognición social, el carácter adaptativo del pensamiento, la carga cognitiva, el aprendizaje situacional o la flexibilidad cognitiva $(7,10)$. La tabla 4 presenta un compendio de las teorías de las ciencias cognitivas y del aprendizaje a las que hacen referencia los documentos incluidos en esta revisión.

\section{Tabla 4}

Visión general de las teorías de las ciencias cognitivas y del aprendizaje

\begin{tabular}{|c|c|c|}
\hline Teoria & Siglas & Decripcion \\
\hline $\begin{array}{l}\text { Teoria del caracter adaptativo del } \\
\text { pensumiento }\end{array}$ & ACT-R & 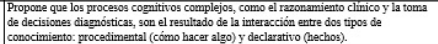 \\
\hline Teoria de la carga cognitiva & CLT & 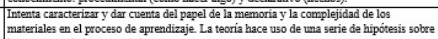 \\
\hline Teoria del aprendizaje sitracional & & 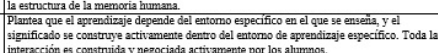 \\
\hline Teeria de la fexibilidad cognititia & CFT & 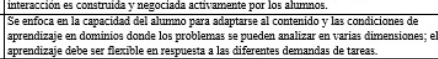 \\
\hline Teoria social-cognitiva & & 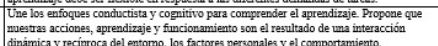 \\
\hline
\end{tabular}

Fuente: elaboración propia a partir de $(6,9)$.

En los últimos años, la tendencia en las reformas curriculares de los programas de pregrado de medicina ha sido la de diseñar e implementar currículos que promuevan mayor integración del conocimiento, inclusión de cursos interdisciplinarios donde prime la experiencia clínica temprana, la enseñanza en grupos pequeños y el énfasis en el desarrollo de habilidades, destrezas y competencias (tabla 5). Los enfoques curriculares que predominan actualmente son los currículos basados en resultados o en competencias. El modelo de la Universidad de Dundee es un ejemplo del currículo basado en resultados y el que implementó la Universidad de Brown es un buen ejemplo de un CBC $(13,21,22,23)$.

\section{Tabla 5}

Cambios en las características curriculares de los programas de medicina

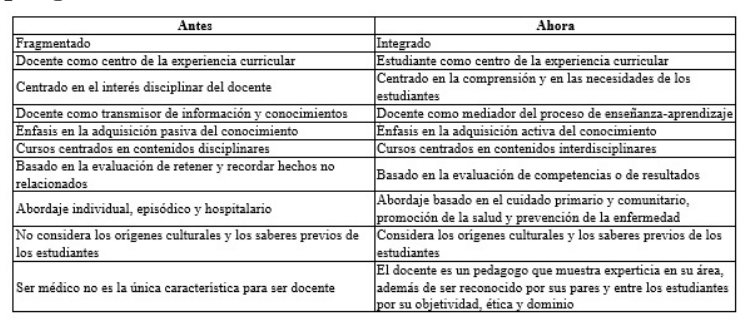

Fuente: elaboración propia a partir de $(7,10,11,12,21,23,26,27,31,34)$.

\section{Aprendizaje}

De los 34 artículos revisados, el 54,3\% aborda el tema del aprendizaje, y de estos, el 21,0\% hace referencia a su definición; el $42,1 \%$, a los 
métodos; el 15,8\%, a la motivación, y el 21,1\%, a los resultados de aprendizaje. El aprendizaje se define como el proceso por el cual las personas adquieren conocimientos, destrezas, actitudes y comportamientos a lo largo de la vida $(7,10)$. Cuando este aprendizaje se orienta al desarrollo de competencias y al alcance de la experticia en un dominio, deberán darse procesos formales de educación y entrenamiento (10). Por su parte, los resultados de aprendizaje hacen referencia a lo que se espera sea alcanzado por los estudiantes durante el proceso de formación $(20,24,25)$. En ese sentido, los resultados de aprendizaje están interconectados, son progresivos y se van adaptando a las diferentes fases del currículo (13).

En los programas de pregrado de medicina, el proceso de aprendizaje se orienta a que los estudiantes desarrollen los conocimientos, las habilidades, las destrezas, los valores y las actitudes para el ejercicio completo y seguro de las actividades médicas (10).

En concordancia con lo anterior, han sido acogidos diferentes métodos de enseñanzaaprendizaje para los programas de pregrado de medicina, entre los que sobresalen el aprendizaje basado en problemas (ABP) y el aprendizaje basado en resultados (ABR) $(10,20)$. En ambos se busca fortalecer la autonomía y la participación de los estudiantes, pero el ABP pone el relieve en el desarrollo de habilidades para la resolución de problemas; mientras que el ABR se orienta hacia el logro de resultados específicos $(7,14,20$, 23, 26, 27, 28). La Universidad de Dundee es un ejemplo de un programa de formación médica que usa el ABP; entre tanto, la Universidad de New South Wales, en Australia, es un ejemplo de una institución educativa que ha adoptado el ABR.

\section{Evaluación}

De los 34 artículos revisados, el 91,4\% hace referencia a la categoría de evaluación, y de esta, el 28,1\%, a su definición; el 46,9\%, a los métodos; el 9,4\%, a la definición de retroalimentación, y el 15,6\%, a los métodos de retroalimentación.

La evaluación es un proceso holístico que incluye el aporte de las experiencias de los docentes, de los estudiantes, de los directivos, de los administrativos y de los egresados, al igual que la definición de indicadores de monitoreo para dar seguimiento a la implementación del plan curricular y garantizar el mejoramiento continuo (27). La evaluación también se usa para documentar el logro y desempeño de aprendizajes, la toma de decisiones administrativas, la promoción del profesorado y el reconocimiento de la excelencia en la educación médica $(10,27)$. La tabla 6 muestra las cinco premisas fundamentales de la evaluación.

\section{Tabla 6}

Cinco premisas de la evaluación

\begin{tabular}{|l|}
\hline Premisas \\
\hline $\begin{array}{l}\text { La evaluación es parte imprescindible en el proceso enseñanza- } \\
\text { aprendizaje. }\end{array}$ \\
\hline $\begin{array}{l}\text { La evaluación debe utilizar diferentes técnicas y estrategias, así como } \\
\text { aplicarse en contextos diversos. }\end{array}$ \\
\hline $\begin{array}{l}\text { La evaluación debe tener criterios explícitos e incluir los momentos de } \\
\text { retroalimentación y autoevaluación. }\end{array}$ \\
\hline $\begin{array}{l}\text { La evaluación debe ser tanto formativa como sumativa, y estas pueden } \\
\text { ocurrir en ambientes reales o simulados de aprendizaje. }\end{array}$ \\
\hline La evaluación debe ser un proceso continuo y sistemático. \\
\hline
\end{tabular}

Fuente: adaptado de $(9,17)$.

La evaluación debe ser tanto formativa como sumativa, y esta puede ocurrir en ambientes reales o simulados de aprendizaje y debe combinar calificación numérica, con criterios cualitativos y descriptivos, lo cual se puede esquematizar en una rúbrica $(10,16,21,26,27$, 29, 30). El autoaprendizaje, la autoevaluación y la coevaluación son estrategias fundamentales en los procesos de evaluación (31).

La retroalimentación es el eje de la evaluación, es vital para el aprendizaje y es la esencia de la educación médica. Hattie y Timperley, citados en Norcini y Burch (30), la han definido como "[la] información dada por un agente (profesor, par, uno mismo, etc.) en relación con los aspectos del desempeño o la comprensión de algo" (p. 862) para lo cual se deben considerar factores cognitivos y no cognitivos. La retroalimentación debe estar acompañada siempre de comentarios narrativos y detallados de cómo el estudiante 
debe mejorar para el siguiente avance en el proceso de ser evaluado. La retroalimentación implica juicios que pueden fragmentar y hacer más distante la relación entre el docente y el estudiante, por lo cual debe ser siempre constructiva, oportuna, gradual y a largo plazo $(10,29,30)$.

Para evaluar competencias es importante usar criterios descriptivos, que sean claros tanto para los docentes como para los estudiantes, y que estos sean consistentes en su forma de uso. La tabla 7 resume las principales características de la evaluación basada en competencias.

\section{Tabla 7}

Características de la evaluación en un currículo

basado en competencias

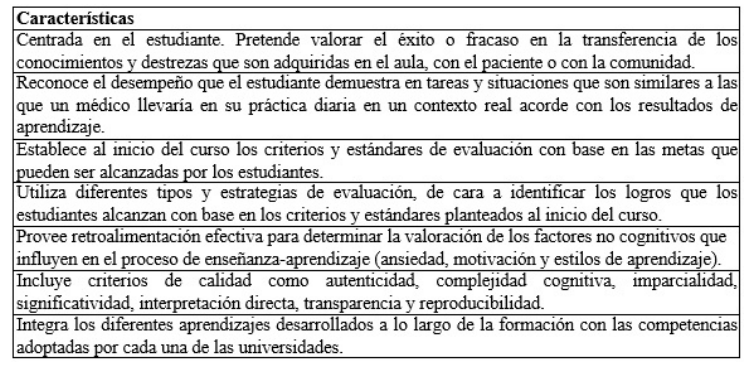

Fuente: elaboración propia a partir de $(6,10,16,21,32,33)$.

\section{Conclusiones}

El enfoque de la educación basada en competencias cada vez se utiliza más en la educación superior, especialmente en países del hemisferio norte; sin embargo, su aplicación en el ámbito médico es un proceso que comenzó a desarrollarse con fuerza en las últimas décadas. En el caso de América Latina, este enfoque hasta ahora se está implementando sin tener todavía literatura que evidencie las repercusiones de sus desarrollos y avances.

La revisión nos permite concluir que, a pesar de que no existe un consenso sobre qué es competencia, $\mathrm{CBC}$ y evaluación por competencias, el optar por un $\mathrm{CBC}$ en un programa de pregrado de medicina, implica reconocer el contexto social y regulatorio donde se aplique, al igual que el análisis de las necesidades sociales de la población donde los profesionales pondrán en práctica sus competencias para transformar los procesos de salud-enfermedad de sus comunidades.

Qué es y qué entendemos por competencia depende, entonces, de las circunstancias políticas, sociales y económicas; de las necesidades de salud; de la disponibilidad de recursos, y de la estructura del sistema de cuidado en el que se construya y se use dicha definición $(10,32)$; sin embargo, existen dos aspectos inherentes a la competencia: el primero es su relación con la práctica para y en la vida real $(9$, 24) y el segundo es su énfasis en el producto final del aprendizaje $(9,13)$.

A partir de esta revisión, nosotras ampliamos el significado de competencia y consideramos que, en el ámbito médico, el concepto no se puede reducir a un único momento de la formación, pues ser competente implica, además de lo mencionado, fortalecer el potencial para resolver problemas, ser crítico y pensar reflexivamente; también que los conocimientos, habilidades y destrezas sean puestos en práctica en diferentes escenarios (aula, hospital, centros de salud, comunidad, entre otros) en el desempeño cotidiano de su oficio, así como en el ser íntegro y ético en cada una de las dimensiones de la vida.

Las competencias son, así, un concepto complejo y cambiante que se adecua a las necesidades y contextos; por ende, en el marco de una reforma curricular y de un proceso de formación, se requiere pensar y discutir constantemente sobre cómo su implementación afectará el perfil del estudiante, la didáctica, la evaluación, el modelo pedagógico, entre otros determinantes del hacer pedagógico que tienen una incidencia en lo que distingue y orienta el hacer y ser de cada programa.

De acuerdo con Patel et al. (10), cuando un programa de formación se estructura para el desarrollo de competencias, es porque existe una referencia o estándar específico que indica los conocimientos y los resultados de aprendizaje en un dominio determinado. Algunos autores afirman que en la actualidad las competencias hacen parte de los criterios médicos de formación. Así, en el marco de un CBC, deben establecerse criterios claros para su identificación 
y creación, a fin de generar, por una parte, mayor regulación en la formación médica (9); por otra, identificar las características, las capacidades y los conocimientos esperados en estos profesionales (32), en la medida en que estas competencias orientan el diseño y puesta en marcha del programa curricular.

La adquisición de competencias es, por lo tanto, un proceso gradual en la formación médica, donde es esencial un equipo docente y un grupo directivo que esté dedicado al diseño, a la implementación, a la evaluación y al seguimiento de un CBC. El éxito de la implementación de un $\mathrm{CBC}$ está en la comprensión consensuada de qué se entiende por competencias, en la definición de los resultados de aprendizaje y en el establecimiento de criterios para su evaluación.

En este sentido, nosotras concluimos que un $\mathrm{CBC}$ en el área médica se encarga de definir los conocimientos, las habilidades y las destrezas que están por adquirir los sujetos en formación en cuatro niveles de competencia: saber qué, saber cómo, saber hacer. saber ser. Esto hace necesario que desde el principio del proceso se conozcan los resultados de aprendizaje, tanto por los estudiantes como por los docentes, lo que facilita los acuerdos y las dinámicas que se gestionan en los diferentes momentos de la formación en los programas de pregrado de medicina.

Adicionalmente, la integración de las ciencias básicas, clínicas y sociales supone un reto que se orienta hacia el aprendizaje activo de los estudiantes. En este escenario, el rol de los docentes debe ser de gestores de la integración no solo de los contenidos propuestos en las asignaturas, sino disciplinar y transdisciplinariamente en el constante diálogo con sus pares académicos y profesionales.

En este punto, queremos hacer algunas precisiones sobre las características de la evaluación en el CBC, considerando que siempre debe: 1) capturar y evaluar el progreso del desempeño que el estudiante muestra en tareas y situaciones similares a las que un médico enfrentaría en su práctica diaria en un contexto real acorde con los resultados definidos de aprendizaje; 2) tener una retroalimentación efectiva que evalúe factores no cognitivos (ansiedad, motivación y estilos de aprendizaje); 3) ser una evaluación centrada en el estudiante y que permita transferir los conocimientos y destrezas del aula al paciente o a la comunidad $(6,10,13,21,32,33) ; 4)$ establecer indicadores posibles de ser alcanzados por los estudiantes; 5) utilizar diferentes actividades de evaluación que puedan reflejar los mismos indicadores, y 6) garantizar que las tareas de evaluación se encuentran alineados con la complejidad cognitiva del aprendizaje, no ser ni más complejos ni más simple que el resultado de aprendizaje propuesto desde el inicio del curso (6).

Finalmente, queremos subrayar que la retroalimentación hace parte de la evaluación y promueve el aprendizaje de tres maneras: 1) informando a los estudiantes su progreso o la falta de este, 2) orientando a los estudiantes a partir de las necesidades de aprendizaje observadas y los recursos disponibles para facilitar su aprendizaje y 3) motivando a los estudiantes a comprometerse en actividades de aprendizaje apropiadas (30). Un factor que marca la diferencia en cómo la retroalimentación incide en el proceso de aprendizaje es la credibilidad que dan los estudiantes de quién hace la retroalimentación (10).

\section{Recomendaciones}

Al haber optado por un $\mathrm{CBC}$ se recomienda:

Establecer un marco de referencia basado en los modelos proporcionados por las teorías cognitivas.

Asumir una definición de competencias que permita la alineación del currículo, los métodos de enseñanza, la evaluación y la formación docente.

Considerar la evaluación como un proceso dinámico y complejo que orienta los esfuerzos del aprendizaje y no simplemente como una labor instrumental. 


\section{Conflicto de intereses}

Las autoras declaran no tener ningún conflicto de intereses para la realización del presente artículo.

\section{Agradecimientos}

Las autoras desean agradecer a María Fernanda Parra Murillo, por su valiosa colaboración con la edición de contenido del artículo.

\section{Referencias}

1. Boucher A, Frank JR, Van Melle E, Oandasan I, Touchie C. Competency based medical education: a white paper commissioned by the AFMC board of directors [internet]; 2017. Disponible en: https://mededconference.ca/sites/d efault/files/AFMC-CompetencyBased

MedicalEducation_en.pdf

2. Frank JR, Mungroo R, Ahmad Y, Wang M, De Rossi S, Horsley T. Toward a definition of competency-based education in medicine: a systematic review of published definitions. Med Teach. 2010;32(8):631-7. https://doi.o $\mathrm{rg} / 10.3109 / 0142159 X .2010 .500898$

3. Peters MDJ, Godfrey CM, Mcinerney P, Soares CB, Khalil H, Parker D. Methodology for JBI Scoping Reviews. Joanna Briggs Insitute. 2015;53(9):0-24. https://doi.org/10.10 17/CBO9781107415324.004

4. Thomas A, Lubarsky S, Durning SJ, Young ME. Knowledge syntheses in medical education: demystifying scoping reviews. Acad Med. 2017;92(2):161-6. https://doi.org /10.1097/ACM.0000000000001452

5. Moher D, Liberati A, Tetzlaff J, Altman DG. Preferred reporting items for systematic reviews and metaanalyses: the PRISMA statement. Int J Surg. 2010;8(5):336-41. https://doi.org /10.1016/j.ijsu.2010.02.007
6. Valverde Berrocoso J, Revuelta Domínguez FI, Fernández Sánchez MR. Modelos de evaluación por competencias a través de un sistema de gestión de aprendizaje. Rev Iberoam Educ [internet]. 2012;60(60):51-62. Disponible en: http://dialnet.unirioja.e s/servlet/articulo? codigo $=4772438 \& i$ $\mathrm{nfo}=$ resumen\&idioma $=\mathrm{ENG}$

7. Goldie J. Integrating professionalism teaching into undergraduate medical education in the UK setting. Med Teach. 2008;30(5):513-527. https://doi .org/10.1080/01421590801995225

8. Harden RM. Learning outcomes as a tool to assess progression. Med Teach. 2007;29(7):678-682. https://doi.org/10 $.1080 / 01421590701729955$

9. Albanese MA, Mejicano G, Mullan P, Kokotailo P, Gruppen L. Defining characteristics of educational competencies. Med Educ. 2008;42 (3):248-255. https://doi.org/10 $.1111 / j .1365-2923.2007 .02996 . x$

10. Patel VL, Yoskowitz NA, Arocha JF. Towards effective evaluation and reform in medical education: a cognitive and learning sciences perspective. Adv Heal Sci Educ. 2009;14(5):791-812. https://doi.org/10 $.1007 / \mathrm{s} 10459-007-9091-1$

11. Shumway JM, Harden RM. AMEE guide no. 25: the assessment of learning outcomes for the competent and reflective physician. Med Teach. 2003;25(6):569-84. https://doi.org/10. 1080/0142159032000151907

12. Harris DL, Krause KC, Parish DC, Smith MU. Academic competencies for medical faculty. Fam Med. 2007;39(5):343-50. https://doi.org/10. $1002 / \mathrm{smj}$

13. Harden RM, Crosby JR, Davis MH. AMEE guide No. 14: outcome-based education: Part 1 - An introduction to outcome-based education. Med Teach. 
1999;21(1):7-14. https://doi.org/10.10 80/01421599979969

14. Dolmans D, Schmidt $H$. The advantages of problembased curricula. Postgrad Med J. 1996;72 (851):535-8. https://doi.org/10 $.1136 /$ pgmj. 72.851 .535

15. Litzelman DK, Cottingham AH. The new formal competency-based curriculum and informal curriculum at Indiana University School of Medicine: overview and five-year analysis. Acad Med. 2007;82(4):410-21. https://doi.or g/10.1097/ACM.0b013e31803327f3

16. Derstine PL. Implementing goals for non-cognitive outcomes within a basic science course. Acad Med. 2002;77 (9):931-2. https://doi.org/10.1 097/00001888-200209000-00035

17. Cottingham AH, Suchman AL, Litzelman DK, et al. Enhancing the informal curriculum of a medical school: a case study in organizational culture change. J Gen Intern Med. 2008;23(6):715-22. https://doi.org/10. 1007/s11606-008-0543-y

18. Suchman AL, Williamson PR, Litzelman DK, Frankel RM, Mossbarger DL, Inui TS. Toward an informal curriculum that teaches professionalism. J Gen Intern Med. 2004;19(5):501-4. https://doi.org/10.1 111/j.1525-1497.2004.30157.x

19. Howe A. Twelve tips for developing professional attitudes in training. Med Teach. 2003;25(5):485-7. https://doi.o $\mathrm{rg} / 10.1080 / 01421590310001605624$

20. Harden RM. AMEE guide No. 21: Curriculum mapping: a tool for transaprent and authentic teaching and learning. Med Teach. 2001;23(2):123-37. https://doi.org/10. 1080/01421590120036547

21. Smith SR, Dollase RH, Boss JA, Assessing students' performances in a competency- based curriculum. Acad Med. 2003;78(1):97-107. https://doi.org/10. 1097/00001888-200301000-00019

22. Harden RM. The integration lader: a tool for curriculum planning and evaluation. Med Educ. 2000;34:551-557. https://doi.org/10.10 46/j.1365-2923.2000.00697.x

23. Jones R, Higgs R, Angelis Cde, Prideaux D. Changing face of medical curricula. Lancet. 2001;357(9257):699-703. https://doi.o $\mathrm{rg} / 10.1016 / \mathrm{S} 0140-6736(00) 04134-9$

24. Díaz A. Construcción de programas de estudio en la perspectiva del enfoque de desarrollo de competencias. Perfiles Educ. 2014;XXXVI(143):142-62.

25. Cumming A, Cumming A, Ross $\mathrm{M}$. The tuning project for medicine learning outcomes for undergraduate medical education in Europe. Med Teach. 2007;29(7):636-41. https://doi. org/10.1080/01421590701721721

26. Davis MH, Harden RM, AMEE medical education guide No. 15: problem-based learning: a practical guide. Med Teach. 1999;21(2):130-40. https://doi.org/10. 1080/01421599979743

27. Gibson KA, Boyle P, Black DA, Cunningham M, Grimm MC, McNeil HP. Enhancing evaluation in an undergraduate medical education program. Acad Med. 2008;83(8):787-93. https://doi.org/10. 1097/ACM.0b013e31817eb8ab

28. Finucane P, Nicholas T, Prideaux D. The new medical curriculum at Flinders University, South Australia: from concept to reality. Med Teach. 2001;23(1):76-9. https://doi.org/10.10 80/0142159002005640

29. Pugh D, Regehr G. Taking the sting out of assessment: is there a role for progress testing? Med Educ. 
2016;50(7):721-9. https://doi.org/10.1 111/medu.12985

30. Norcini J, Burch V. Workplacebased assessment as an educational tool: AMEE guide No. 31. Med Teach. 2007;29(9-10):855-71. https://doi.org/ 10.1080/01421590701775453

31. Al Kadri HM. Redesigning an educational assessment program. Saudi J Kidney Dis Transpl. 2009;20(3):476-80.

32. Durante E. Algunos métodos de evaluación de las competencias: escalando la pirámide de Miller. Rev Hosp Ital BAires [internet]. 2006;26(2):55-61. Disponible en: http: //campus.hospitalitaliano.org.ar/plugin file.php/288499/mod_page/content/6/ Durante_2006.pdf

33. Harris P, Snell L, Talbot M, Harden RM. Competency-based medical education: Implications for undergraduate programs. Med Teach. 2010;32 (8):646-50. https://doi.org/10. 3109/0142159X.2010.500703

34. Maudsley G. The limits of tutors' comfort zones with four integrated knowledge themes in a problem-based undergraduate medical curriculum (Interview study). Med Educ. 2003;37(5):417-23. https://doi.o rg/10.1046/j.1365-2923.2003.01497.x 